\title{
From Syphilis to Autism, How the Anti-Vaccination Movement of Today is an Echo of the Past
}

\author{
Kayleigh Beaveridge ${ }^{1}$
}

${ }^{1}$ McGill University, Montreal, Canada

\section{Correspondence}

Kayleigh Beaveridge

Email: kayleigh.beaveridge@mail.mcgill.ca

Publication Date

January 04, 2021

MJM 2021 (19) 1

\section{McGill}

\section{Journal of Medicine}

www.mjmmed.com

\section{c) (1) (2)}

This work is licensed under a Creative Commons BY-NC-SA 4.0 International License.

\section{ABSTRACT}

Introduction: The anti-vaccination movement has led to decreased vaccination rates and increased vulnerability to vaccine-preventable diseases in the general population. In order to better understand the antivaccination movement of today, the anti-vaccination movement that emerged in the 19th century is examined and measured against the one observed in the 20th century. Discussion: Though the population of the 19 th and 20th centuries differ in many regards and our knowledge of vaccine and immune mechanisms are far greater; the anti-vaccination movement seen today stands on the same pillars as that of the 1800 s with the sentiment of fear at its core. Though the façade of these pillars has been altered to suit the world today, both movements exploited the influence of prominent public figures, maintained false associations with dire vaccine consequences and emphasized these through the use of visual media, repetition and personal narratives. The persistence of the antivaccination movement lies largely in the use of personal stories which are more impactful and memorable then the statistical characteristics of scientific study. Conclusion: The pro-vaccination movement must respond to the tactics used by the anti-vaccination movement and create accessible, understandable and equally impactful communication strategies in order to prevent the spread of misinformation and counter the efforts of the current anti-vaccination movement. Relevance: Vaccine hesitancy was listed amongst the top 10 global health threats in 2019 by the World Health Organization. In order to shift the negative rhetoric surrounding vaccines, the anti-vaccination movement of today and its historic roots need to be understood.

\section{KEYWORDS}

Anti-vaccination, Dr. Edward Jenner, Vaccine Hesitancy, Global Health 
Vaccinations, like many other medical interventions, rely heavily on public opinion for their success. Regardless of the positive or consistent results demonstrated through precise scientific study, if the public does not believe in the efficacy or safety of these interventions then they will fail. Hence, an understanding of public opinion and controversy is key to increasing compliance and acceptance of the intervention in question. From the start, vaccination was rife with controversy within the medical field which spilled out into the lay discourse and set the groundwork for the anti-vaccination movement (1). Despite advances in knowledge related to vaccine mechanism of action, vaccine targets, and immune system responses the anti-vaccination movement persists. Regardless of increased health literacy and increased education in the general population, individuals remain susceptible to the influence of misinformation and fear spread by the anti-vaccination movement. The ability of this movement to influence public practice is evident in the falling rates of vaccine compliance which has left people susceptible to vaccine-preventable diseases and led to outbreaks of diseases like measles in several western countries (2). The consequences of this movement have contributed to the World Health Organization (WHO) listing vaccine hesitancy among the top 10 public health threats in January 2019 (3). It is therefore imperative that health care professionals have an understanding of the movement and its strategies in order to address the issue, if and when, it should arise in the clinical context. In this paper we will take a glimpse at the past in an effort to understand the present and shed light on just how the anti-vaccination movement has persisted all these years. The consequences of the movement in present-day society will then be discussed, as well as, some of the current efforts being made to mitigate the consequences of the anti-vaccination discourse at the level of individuals and the larger community.

\section{1 | THE BIRTH OF ANTI- VACCINATION IN THE 19TH CENTURY}

In 1801, Dr. Edward Jenner published On the Origin of Vaccine Inoculation in which he described his observations that individuals previously exposed to cowpox did not develop smallpox when exposed to the disease (4). He went on to hypothesize that inoculation with cowpox would lead to sustained immunity against smallpox and supported this with descriptions of his experimentation and success (4). One key characteristic of Jenner's new technique was that those inoculated were not contagious, and therefore posed no threat to those around them (1). Another important aspect of the vaccine was its scalability. Given that the vaccine material could be cultured, dried, transported, and re-animated for later use it was possible to reach a large population of people in vast areas (1) The propagation of the vaccine was later made even easier when it became possible to culture the lymph of previously vaccinated individuals and extract the necessary vaccine material (1) Thus, Dr. Edward Jenner had just demonstrated a safe method, without the risk of contagion that could help stop the spread of smallpox, the disease responsible for $10-20 \%$ of burials in urban British cities in the 18th century (5). Yet, rather than being met with praise Jenner was faced with strong opposition from peers and the public alike.

At the time of Jenner's discovery, the accepted practice for smallpox prevention was variolation (1). Variolation involved infecting individuals with matter from the pustules of individuals infected with smallpox (6). Though this technique showed some promise, it was responsible for the death of approximately $2 \%$ of variolated patients and held the drawback that those inoculated became highly contagious themselves; thus, posing a significant risk to others (1). This technique, though hazardous, was supported by influential British physicians such as Dr. Moseley and Dr. Rowley, who largely gleaned their livelihood from its administration (1). The advent of vaccination was perceived as a threat to the variolation business and thus became the target of scorn and misinformation on the part of Dr. Mosley, 
Dr. Rowley and others in the medical field. These physicians successfully led the campaign to skew the vaccine discourse from a medical success story to one wrought with deceit; instilling fear and doubt in the public and associating vaccines with public taboos like bestiality and venereal disease (1). This was achieved through the use of caricatures and images in booklets that were distributed to the public as early as 1802 (1). One such caricature, titled The Cow-Pock (see Figure 1), depicts a scene in which Dr. Jenner administers his vaccine to a woman while surrounded by a mass of individuals in various degrees of distress as they grow horns, birth a bull-like creature, or have cow-shaped growths sprout from their body. Though far from being proof that Jen-

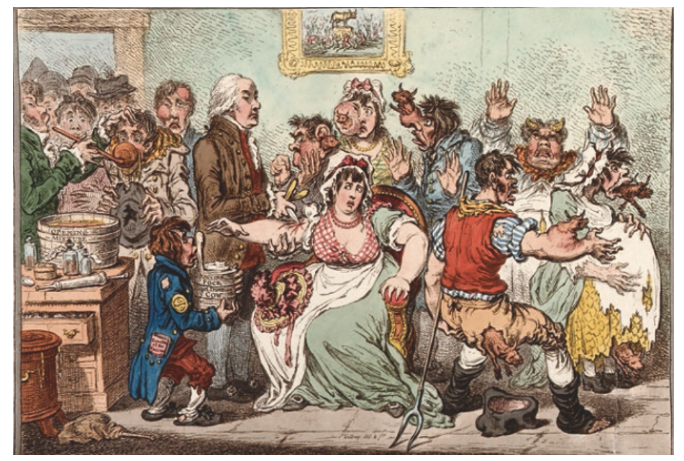

FIG URE 1 "The Cow-Pock" by James Gillray, 1802 (Wellcome Library, London). Patients developing horns or cow-shaped growths following administration of smallpox vaccine.

ner's vaccine led to such consequences, these caricatures were enough to associate vaccination with bestiality and influence public opinion on the morality of vaccines (1). These caricatures were closely followed by drawings, published by Dr. Mosley and based on the findings of Dr. Rowley, depicting individuals who developed animal-like features following the inoculation with the smallpox vaccine (see Figure 2) (1). These drawings were an important step in solidifying the growing fear regarding the consequences of vaccines. By publishing verisimilar drawings of animal-like people, the anti-vaccination movement bluntly implied that what happened to the subjects of these drawings could, and

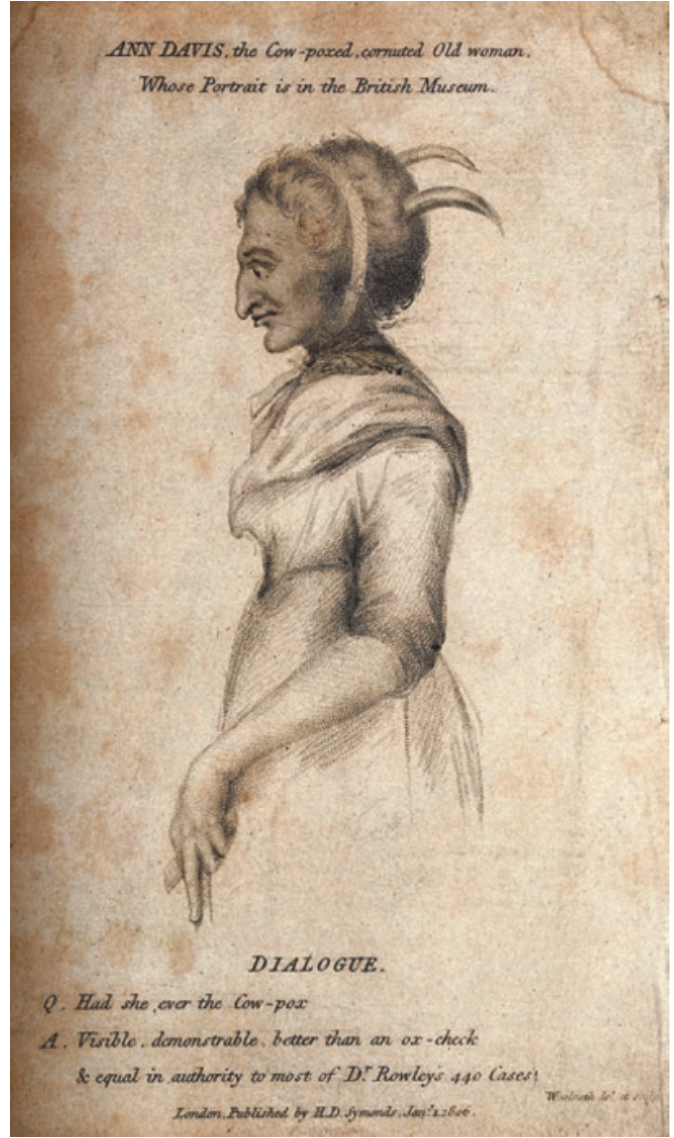

FIG URE 2 Drawing of Ann Davis, 1806 (Wellcome Library, London). Depiction of patient who developed horns after being vaccinated against smallpox.

would, happen to any regular person who received the new smallpox vaccine.

In addition to its association with bestiality, the smallpox vaccine soon came to be associated with syphilis through the careful suggestions of prominent physicians like Dr. Moseley (1). The lack of knowledge regarding the mode of transmission of syphilis and the mechanism of action of vaccines, compounded with the authority these figures held, allowed for these unfounded suggestions to be considered fact (8). Hence, smallpox vaccines became linked to syphilis and the anti-vaccination movement had one more argument against vaccines.

The clergy soon joined these physicians in the ranks 
of the early anti-vaccination movement. The Catholic Church opposed vaccination on the basis that it involved injecting people with animal matter which threatened the sanctity of the human body (6). In addition, the Church maintained the belief that it was God's decision whether the suffering of the devoted was to be ended or not. Hence, interfering with God's choice through interventions like vaccination, was disrespectful to $\mathrm{Him}$ (6).

The battle between the pro and anti-vaccination movements of 19th century England came to a head in 1853 when the first law making vaccination compulsory in the first three months of life came into effect (7). Vaccination became a political issue and led to the foundation of official anti-vaccination groups, such as the Leicester Anti-Vaccination League, in 1869 (7). Following escalating protests and public opposition to vaccine laws, the British government launched the Royal Commission on Vaccination which concluded in 1897. One of the myths the Commission targeted was the fear that the smallpox vaccine could infect individuals with syphilis. Although the Commission found that there was no evidence that the smallpox vaccine had caused individuals to become infected with syphilis, this public fear, along with the other myths that had been propagated since the birth of Dr. Jenner's vaccine, persisted (1).

Between the voices of influential physicians, the warnings of the Church, the propagation of frightening images of metamorphosis and the association with syphilis, Dr. Jenner and his vaccine faced great challenges in gaining public trust and the anti-vaccination movement continued to grow.

\section{2 | THE ANTI-VACCINATION MOVEMENT OF THE 21ST CEN- TURY}

Since the 19th century, the pillars upon which the antivaccination movement was supported have largely been eliminated; physicians, and prominent health organizations like the WHO support vaccination, the Catholic Church holds that followers have a moral responsibility to vaccinate, and the mechanism of action of vaccines with their subsequent immune responses are better understood such that concerns over metamorphosis and syphilis have been eliminated $(9,10)$. In addition, smallpox was officially eradicated in December 1979 following a global vaccination campaign led by the $\mathrm{WHO}$, thus demonstrating the efficacy of the vaccine and the potential of the technique (11). Despite these advances in knowledge and the proven success of vaccination, the anti-vaccination movement remains present, influential, and widespread in the 21st century.

The success of the anti-vaccination movement in getting their ideas to the public, for getting their ideas heard, repeated, and easily accessible is largely attributed to the interplay between two factors: decreasing trust in institutional medicine and increasing digital connectedness in the Western world (12). Over the past 50 years, studies have found a significant decline in the confidence of the public towards the medical system (13). During this same period, there has been a surge in the amount of health information accessible to the patient population. Additionally, societal values have shifted the patient-physician relationship from a paternalistic relationship to a model of shared decision making in which patients expect to be given a voice in their medical care (12). The combination of these changes has led the patient population to seek medical information outside of the traditional medical system, often using the Internet as their first and main source. Hence, similarly to how patients in the 19th century distrusted Dr. Jenner because of the presence of contradictory information from seemingly reliable sources, many patients of the 21st century have come to distrust physicians due to an excess of health information that is contradictory, ever-changing and without distinction between accurate or misleading.

The anti-vaccination movement has taken advantage of this new platform to propagate their message instilling doubt and fear regarding vaccination in a growing faction of our population. A closer examination of the rhetoric used today shows a degree of continuity between the anti-vaccinators of the 19th and 21st centuries. In other words, the anti-vaccination movement we know today stands on the same pillars as that of that 
19th century but has simply altered the façade of these pillars to suit the media of the time. Hence, fear mongering remains at the forefront of the movement, but instilling fear and doubt in individuals about vaccines is now achieved with modernized tactics and reflects the fears of our society.

Prominent physicians have been replaced with important public figures in the ranks of celebrities including Jenny McCarthy, Robert De Niro, and political leaders including President Donald Trump (2).

Fear of the development of syphilis and animalistic metamorphosis have been replaced with fears of the development of autism, immune disorders, allergies and sudden infant death syndrome (SIDS) (14). The association, in particular, between the measles, mumps, and rubella (MMR) vaccine and autism is one of the most frequent concerns cited by parents when deciding whether or not to vaccinate their children (15). MMR, as a causative agent of autism, became popularized in the 1990 s following the publication of Dr. Wakefield and colleagues' article in the Lancet, which claimed to have evidence of an association between the MMR vaccine and "chronic enterocolitis and developmental regression" (16). Though the methodology of the study was questioned, its results disproven, and the paper retracted, the association remains at the forefront of the vaccination debate (17). The MMR vaccine-autism association is reminiscent of the smallpox vaccine-syphilis association of the 19th century by its consequences on the population despite a lack of supportive evidence. In both cases, the words of a physician coupled with repetition through visual media and by prominent figures have had a strong impact on the general population.

Caricatures published in booklets and handed out on street corners have been digitalized and bolstered by memes and pictures propagated by social media platforms such as YouTube, Instagram, Facebook, and Tumblr. A study on the presence of anti-vaccination content on YouTube in 2019 found that $32 \%$ of videos about vaccination were against the practice and that these videos had more views and higher ratings then content supporting vaccination (2). Quick searches on other social media platforms yielded caricatures which perpet- uated beliefs that vaccines cause autism or SIDS (see Figures 3 and 4). In addition to these platforms, anti-

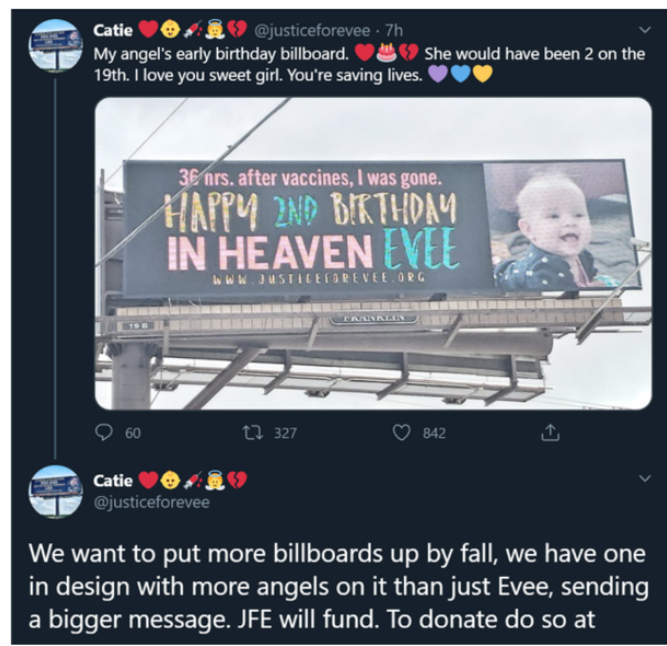

FIGURE 3 Billboard sponsored by Anti-Vaccination movement and posted on Twitter.

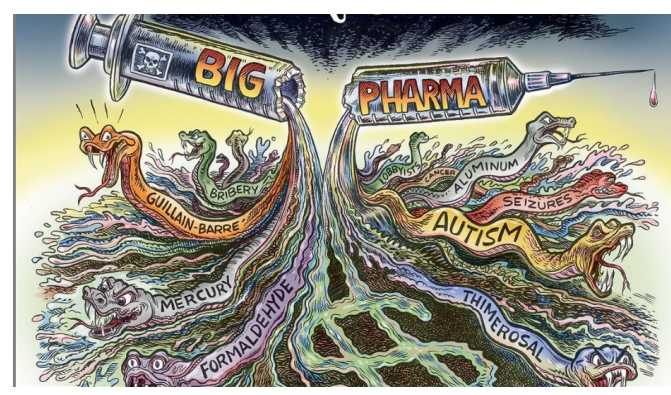

FIGURE 4 Caricature found on Instagram and posted on anti-vaccination page. Suggests that vaccines are controlled by Big Pharma and makes associations between vaccines and autism, seizures, bribery and money.

vaccination groups have been able to reach a broader audience through carefully constructed websites that emphasize the risks of vaccines and claim a lack of evidence for vaccine safety and efficacy. One of the most effective tools used by anti-vaccination websites today is the use of personal stories as proof of the danger of vaccines (17). Take, for example, Vaccine Choice Canada: 
a prominent anti-vaccination group that has accounts on all social media platforms and an independent website (https://vaccinechoicecanada.com). Though their name suggests a degree of neutrality, the website is predominantly filled with warnings to parents about vaccine risks, and suggestions that parents should not trust the medical establishments or body of research supporting vaccines, which have often been sponsored by provaccine groups like public health departments and vaccine manufacturers (18). In addition to this, there is an emphasis on the sharing of personal stories and an invitation for parents to share their own stories of the adverse effects experienced by their children following routine vaccinations (19). There is an evident absence of pro-vaccination personal stories on these pages. Just like the verisimilar drawings of animalistic metamorphosis had an impact on the people of the 19th century, these personal stories have an impact on parents of today who see their children in the stories of other parents and fear that the same adverse effects that others claim to have witnessed will happen to them and their families. Hence, the decades of scientific study supporting vaccine safety, efficacy, and their benefits are no match for the personal stories that pull at our heart strings.

In sum, vaccines of today face similar challenges to their predecessors; the science of vaccines is measured against the word of prominent and trusted public figures, vaccine efficacy is called into question by unfounded associations with grave consequences, and their opponents' exploitation of fear and emotions is more impactful than the technical communication of empirical scientific research.

The culmination of these strategies has led to growing fear and concern amongst parents who have started to question the vaccine recommendations made by the health departments of their countries. One of the challenges in addressing the anti-vaccination movement lies in the diversity of beliefs held amongst its members. In an attempt to represent this spectrum of beliefs, the WHO coined the term vaccine hesitancy and defined it as "a delay in acceptance or refusal of vaccines despite availability of vaccination services" (12). The growing number of vaccine hesitant parents, particularly regard- ing the MMR vaccine, is evident in the growing number of measles cases worldwide and the reversal of the disease status in the United States, a country where measles was considered eradicated in 2000 (20).

\section{I FACING ANTI-VACCINATION RHETORIC}

Falling vaccination rates and increasing vaccine hesitancy amongst parents are growing public health concerns that must be addressed at the systemic and individual level. Many studies have tried to understand the anti-vaccination movement in an effort to formulate recommendations to counter the negative rhetoric that has plagued vaccines for centuries. From these studies, it seems that the anti-vaccination movement has a cultural advantage and that health professionals are starting from a position of weakness. While anti-vaccinators need only instill an ounce of doubt in the minds of the public to make them question vaccine safety, the provaccination movement is held to a different standard and must prove, beyond a doubt, that vaccines are safe, effective and necessary. Even if science were able to deliver a study meeting all of these demands, the hard reality is that the numbers, careful analysis and statistical characteristics of meticulous scientific study will not change minds without an effective communication strategy that can directly address doubt and risk in plainly understandable language or imagery. In this regard the medical and scientific fields have not been successful for decades. In the case of vaccines, sensational rhetoric and imagery remains a primary cause of vaccine hesitancy. Effective strategies to combat sensationalism and its effects remain elusive. Though the chances of the pro-vaccination movement may sound bleak, there are efforts being made to leverage the strength of science and the power of governments to mitigate the misinformation and limit the fear produced by the antivaccination movement. A recent survey-based retrospective study examining the rise and fall of vaccine confidence in 149 countries between 2015-2019 shows just how much political, religious and environmental fac- 
tors play a role in public confidence in vaccination (21). As the study points out, there is a great need to further examine the interplay between these factors and medical decisions made by individuals (21). When narrowing down a diagnosis and determining an appropriate treatment strategy, physicians are taught to view patients through the lens of the social context in which they exist, but it is becoming increasingly obvious that this social context has a strong impact on whether or not patients seek and trust medical care. Hence, individual physicians and larger public health authorities must continually monitor societal opinions and norms in order to ease the effects these have on vaccine uptake. Vaccines aim to prevent the complications of disease before they can even manifest. The pro-vaccination movement must emulate the very therapy it wishes to promote and take a pro-active preventative approach in addressing vaccine hesitancy.

\section{REFERENCES}

1. Boddice R. Bestiality in a Time of Smallpox: Dr. Jenner and the "Modern Chimera": Philosophical, Cultural, and Historical Perspectives. In Ohrem D, Calarco M, editors. Exploring Animal Encounters. Palgrave Macmillan, Cham; 2018. p. 155-78.

2. Benecke O, DeYoung SE. Anti-Vaccine Decision-Making and Measles Resurgence in the United States. Global Pediatric Health. 2019 Jan;6:2333794X1986294.

3. Roberts, M. Vaccines: Low trust in vaccination "a global crisis." BBC News [Internet]. 2019 Jun 19; Available from: https://www.bbc.com/news/health-48512923

4. Riedel S. Edward Jenner and the History of Smallpox and Vaccination. Baylor University Medical Center Proceedings. 2005 Jan;18(1):21-5.

5. Davenport RJ, Boulton J, Schwarz L. Urban inoculation and the decline of smallpox mortality in eighteenth-century cities-a reply to Razzell: The Economic History Review. 2016 Feb;69(1):188-214.

6. History of Anti-vaccination Movements. The History of Vaccines: and educational resource by the College of Physicians of Philadelphia [Internet]. 2018. Available from: https://www.historyofvaccines.org/content/articles/history-antivaccination-movements

7. WatsonG. The anti-vaccination movement that gripped Victorian England. BBC News [Internet]. 2019 Dec 28; Available from: https://www.bbc.com/news/uk-england-leicestershire-50713991 8. Boddice R. Vaccination, Fear and Historical Relevance. History Compass. 2016 Feb;14(2):71-8.
9. World Health Organization (WHO). Immunization [Internet]. Available from: https://www.who.int/topics/immunization/en/.

10. Note on Italian Vaccine Issue [Internet]. Pontifical Academy for Life. Available from: http://www.academyforlife.va/ content/pav/en/the-academy/activity-academy/note-vaccini.html 11. WHO. Frequently asked questions and answers on smallpox [Internet]. 2016. Available from: https://www.who.int/csr/disease/smallpox/faq/en/

12. McClure CC, Cataldi JR, O'Leary ST. Vaccine Hesitancy: Where We Are and Where We Are Going. Clinical Therapeutics. 2017 Aug;39(8):1550-62.

13. Lynch TJ, Wolfson DB, Baron RJ. A Trust Initiative in Health Care: Why and Why Now? Academic Medicine. 2019 Apr;94(4):463-5.

14. Vaccine Choice Canada. Health Risks [Internet]. Available from: https://vaccinechoicecanada.com/health-risks/

15. Dubé E, Laberge C, Guay M, Bramadat P, Roy R, Bettinger JA. Vaccine hesitancy: An overview. Human Vaccines \& Immunotherapeutics. 2013 Aug 8;9(8):1763-73.

16. Wakefield A, Murch S, Anthony A, Linnell J, Casson D, Malik M, et al. RETRACTED: lleal-lymphoid-nodular hyperplasia, nonspecific colitis, and pervasive developmental disorder in children. The Lancet. 1998 Feb;351(9103):637-41.

17. Bean SJ. Emerging and continuing trends in vaccine opposition website content. Vaccine. 2011 Feb 24;29(10):1874-80.

18. Vaccine Choice Canada.Science Supporting Vaccine Risk [Internet]. Available from: https://vaccinechoicecanada.com/sciencesupporting-vaccine-risk/

19. Vaccine Choice Canada. Personal Stories [Internet]. Available from: https://vaccinechoicecanada.com/personal-stories/

20. The Lancet Child \& Adolescent Health. Vaccine hesitancy: a generation at risk. The Lancet Child \& Adolescent Health. 2019 May;3(5):281.

21. De Figueiredo A, Simas C, Karafillakis E, Paterson P, Larson HJ. Mapping global trends in vaccine confidence and investigating barriers to vaccine uptake: a large-scale retrospective temporal modelling study. The Lancet. 2020 Sep;S0140673620315580. 